Faur, E. Género, diversidad sexual y conciliación familia - trabajo. Contrapuntos entre el derecho de familia y el derecho laboral. Derecho y Ciencias Sociales. Octubre 2018. № 19. (Las familias y el derecho de las familias a dos años de vigencia del Código Civil y Comercial) Pgs 45-62. ISNN 1852-2971. Instituto de Cultura Jurídica y Maestría en Sociología Jurídica. FCJ y S. UNLP

\title{
Género, diversidad sexual y conciliación familia-trabajo. Contrapuntos entre el derecho de familia y el derecho laboral
}

Gender, sexual diversity and work-family balance. Counterpoints between family law and labour law

\section{Eleonor Faur}

\section{Resumen:}

Este artículo analiza el contraste entre la ampliación de los derechos de la población LGTTBI y la sombra maternalista que aún pesa sobre las políticas de armonización familia-trabajo. Recorre los avances en el derecho de familia, a partir del cambio de estatus de las mujeres y la población LGTTBI y explora en qué medida éstos han impactado en el derecho laboral, en particular en las licencias parentales para el cuidado de hijos/as pequeños/as. Argumento que el reconocimiento de derechos civiles de gays, lesbianas y trans coexiste con una legislación laboral anacrónica, que define y distingue responsabilidades en función del género. Todo ello da cuenta de un tramado de lógicas contrastantes en las regulaciones estatales. El derecho de familia, avanzando hacia un sistema de igualdad de derechos, y el derecho laboral, sostenida en una lógica maternalista, que no refleja las transformaciones atravesadas en el derecho de familia. De tal modo, omite el reconocimiento de la población LGTTBI, y refuerza las agudas desigualdades de género y clase.

Palabras claves: Cuidado infantil; derecho laboral; derecho de familia; maternalismo; género; diversidad; LGTTBI.

\begin{abstract}
This paper analyzes the contrast between the revolutionary shift in LGBTTI rights and the maternalistic shadow still cast over childcare-related family policies rights in Argentina. It analyzes family laws highlighting recent developments with regards to the recognition of women and the LGBTTI population as equal rights holders in the realm of the family. Then, it examines labor regulations aimed at working parents with childcare responsibilities, exploring whether or not the enactment of the Egalitarian Marriage Act shifted the consideration of working mothers and fathers' rights and obligations regarding childcare. Finally, it discusses the progress and related challenges - in implementing policies that can disentangle the gender and social inequalities embedded in them. I argue that the breakthrough in LGBTTI rights is situated in the persistent continuity of the sexual division of labor established through labor regulations aimed at working parents. These not only

\footnotetext{
- Profesora de la Universidad Nacional de San Martín, Investigadora del CIS-IDES. Una versión previa de este artículo fue publicada en Faur, E. 2018 "Contrasting trends in gender and childcare in Argentina: Family policies between LGBT rights and maternalism" en Current Sociology Monograph. 1-12. La autora agradece a María Laura González, abogada (UNLP), traducción del texto original en inglés posteriormente modificado y evaluado. Finalmente, se agradece a la abogada Karina Andriola, por el intercambio de ideas. La escritura de este artículo fue posible gracias a la estancia como Profesora e investigadora invitada, Cátedra Ela-Bhatt, ICDD, Universidad de Kassel.
} 
Faur, E. Género, diversidad sexual y conciliación familia - trabajo. Contrapuntos entre el derecho de familia y el derecho laboral. Derecho y Ciencias Sociales. Octubre 2018. № 19. (Las familias y el derecho de las familias a dos años de vigencia del Código Civil y Comercial) Pgs 45-62. ISNN 1852-2971. Instituto de Cultura Jurídica y Maestría en Sociología Jurídica. FCJ y S. UNLP

misrecognize the LGBTTI population, but they also reinforce a system of socioeconomic and inequalities. This scenario reaffirms maternalistic assumptions that do not reflect the new family dynamic and recent legal advances. In addition, in the most unequal region of the world, the need to close socioeconomic gaps is crucial.

Key words: Childcare; Labour Rights; Family Rights; Gender; LGBTTI; Maternalism. 
Faur, E. Género, diversidad sexual y conciliación familia - trabajo. Contrapuntos entre el derecho de familia y el derecho laboral. Derecho y Ciencias Sociales. Octubre 2018. № 19. (Las familias y el derecho de las familias a dos años de vigencia del Código Civil y Comercial) Pgs 45-62. ISNN 1852-2971. Instituto de Cultura Jurídica y Maestría en Sociología Jurídica. FCJ y S. UNLP

\title{
Género, diversidad sexual y conciliación familia-trabajo. Contrapuntos entre el derecho de familia y el derecho laboral.
}

\author{
Eleonor Faur
}

\section{Introducción}

En la Argentina, el cuidado infantil y los arreglos institucionales en torno al mismo se forjaron a partir de una marcada diferenciación de género. Históricamente, las mujeres -en su condición de madres- fueron consideradas las principales proveedoras de cuidados, mientras que los padres permanecieron prácticamente al margen de dicha responsabilidad. Basadas en este patrón general, las políticas tendientes a armonizar las responsabilidades entre familia y trabajo no fueron neutrales en términos de género, sino que definieron como sujeto de esa conciliación a las mujeres (Faur, 2006). Al mismo tiempo, se asentaron en un modelo heteronormativo (Lind y Share, 2003), que excluyó a la población LGTTBI de los derechos asociados con la conformación de vínculos familiares y los beneficios derivados de éstos.

En la última década, el modelo heteronormativo comenzó a erosionarse. La Ley de Matrimonio Igualitario ( $N^{\circ}$ 26.618), sancionada en el año 2010, colocó a la Argentina como el primer país latinoamericano que aprobó el vínculo matrimonial entre personas del mismo sexo. En los años subsiguientes, una serie de normas reconocieron y ampliaron derechos civiles para las lesbianas, gays, trans, travestis, bisexuales e intersexuales (en adelante con las siglas LGTTBI en adelante) ${ }^{1}$. En la actualidad, la legislación en materia de familia reconoce y protege la identidad, el matrimonio y la parentalidad de personas LGTTBI.

¿En qué medida la ampliación de derechos de LGTTBI desafió la distinción de derechos de madres y padres en torno al cuidado y repercutió en una institucionalidad tendiente a la igualdad en los cuidados familiares?. Si entendemos a las políticas de igualdad de género como aquellas que apuntan a desmantelar jerarquías de poder que privilegian a los hombres y a lo masculino; la división sexual del trabajo que devalúa a las mujeres y lo femenino, y la institucionalización de la heteronormatividad (Htun y Weldon, 2010), en teoría, este nuevo ordenamiento legal contribuiría a resquebrajar la demarcación de derechos asignados en función del género. Sin embargo, como advierten Morgan y Orloff los estados muestran "muchas manos, distintas escalas de gobierno y lógicas múltiples y potencialmente contradictorias" (Morgan y Orloff, 2017: 3).

\footnotetext{
${ }^{1}$ La sigla refiere a lesbianas, gays, trans, travestis, bisexuales e intersexuales.
} 
Faur, E. Género, diversidad sexual y conciliación familia - trabajo. Contrapuntos entre el derecho de familia y el derecho laboral. Derecho y Ciencias Sociales. Octubre 2018. № 19. (Las familias y el derecho de las familias a dos años de vigencia del Código Civil y Comercial) Pgs 45-62. ISNN 1852-2971. Instituto de Cultura Jurídica y Maestría en Sociología Jurídica. FCJ y S. UNLP

Este artículo busca explorar las lógicas de género que atraviesan el derecho de familia y el derecho laboral en el campo del cuidado. Para ello, en primer lugar, se presenta un breve marco conceptual. A continuación, se examinan las normas vinculadas a la constitución y disolución del matrimonio y los vínculos entre los miembros de las familias, subrayando las modificaciones que han tenido en relación con el estatus de mujeres y de la población LGTTBI, y el paulatino reconocimiento de la igualdad entre hombres, mujeres, LGTTBI. Posteriormente, examina las normativas del derecho laboral -en particular las que regulan licencias para madres y padres trabajadores/as. Esta indagación repone la pregunta acerca del modo en el cual distintos marcos legales definen sujetos de derechos, y en ocasiones, desarrollan lógicas contrapuestas en términos de género. Finalmente, se presentan algunas consideraciones finales, que subrayan los desafíos pendientes.

2. Políticas públicas, familias y relaciones de género

Como nos recuerda Elizabeth Jelin, los modelos de familia no responden a un formato "natural" sino que son producto de procesos históricos, en los que se ponen en juego las dimensiones productivas y reproductivas de la sociedad, los patrones culturales y los sistemas políticos (Jelin, 2010). El modelo familiar que acompañó a la revolución industrial materializó una marcada distinción de funciones, espacios y actividades según género. A las mujeres se les asignó la responsabilidad central por la reproducción social: el trabajo rutinario e impago en la esfera doméstica y la tarea de criar y cuidar a sus hijos e hijas, mientras que a los hombres se los concibió como los principales responsables de la esfera productiva y, por ende, como los proveedores de ingresos familiares. En la literatura académica, este modelo ha sido referido como el modelo familiar de varón proveedor y mujer ama de casa (Crompton, 1999; Wainerman, 2003). De manera extendida, las mujeres eran concebidas ante todo como madres, y las madres, como "las mejores cuidadoras". Así, el ideal maternalista y la "maternalización de las mujeres" filtraron instituciones, prácticas y representaciones sociales durante largo tiempo, por medio de un conjunto de normativas y políticas públicas afines a esta ideología (Nari, 2004).

En la práctica, este modelo no fue universal: las familias se identificaron con el mismo -o bien, lo desafiaron- de maneras muy diversas (Connell, 1995; Crompton, 2006). No obstante ello, la definición de leyes e instituciones se forjó a partir de un formato de familia y pareja que distinguía sujetos y responsabilidades. Todo ello, asentado en un modelo heteronormativo, que presupone la heterosexualidad como pauta común y extendida (Lind 
Faur, E. Género, diversidad sexual y conciliación familia - trabajo. Contrapuntos entre el derecho de familia y el derecho laboral. Derecho y Ciencias Sociales. Octubre 2018. № 19. (Las familias y el derecho de las familias a dos años de vigencia del Código Civil y Comercial) Pgs 45-62. ISNN 1852-2971. Instituto de Cultura Jurídica y Maestría en Sociología Jurídica. FCJ y S. UNLP

and Share, 2003), y en América Latina se encontró en la base de la creación de los estados nacionales (Pecheny y de la Dehesa, 2010).

Los debates y conceptualizaciones del feminismo lograron correr el velo que naturalizaba la configuración de género sobre la cual se sostenía dicho modelo familiar. Hecharon luz sobre las jerarquías que se encontraban en la base de una organización social que, al mismo tiempo, devaluaba el trabajo reproductivo y de cuidados no remunerado y lo asignaba a las mujeres (Benería, 1979; Larguía y Dumoulin, 1976; Jelin, 2010). Asimismo, revelaron la variedad de maneras en el cual intervinieron los regímenes de bienestar en la construcción de un modelo de familia, trabajo y género, mediante la distinción de derechos y responsabilidades de provisión y cuidados familiares (Lewis, 1997; Sainsbury, 1996, 1999, Martínez Franzoni, 2008).

En las últimas décadas el modelo de varón proveedor/mujer ama de casa se alteró de raíz. En el área metropolitana de Buenos Aires, los hogares con un único proveedor masculino disminuyeron del $74.5 \%$ al $54.7 \%$ entre 1980 y 2000, mientras que los hogares de dos proveedores aumentaron de $25.5 \%$ a $45.3 \%$ (Wainerman, 2003). Por su parte, el reconocimiento de la diversidad sexual y de género permitió hacer visible lo que se encontraba solapado en las dinámicas sociales, invisible en las estadísticas nacionales y discriminado en los sistemas jurídicos. En la Argentina contemporánea, mientras el 38\% del total de los hogares está formado por una pareja heterosexual con hijos, el 11\% corresponde a un hogar monoparental (sobre todo, a cargo de mujeres) y el $1 \%$ a un hogar con pareja del mismo sexo (Binstock, 2018).

En este contexto, especial interés despertó el análisis de (y la abogacía por) las políticas de conciliación entre familia y trabajo y, de manera más general, por las políticas de cuidado -que refieren a intervenciones en las que participan distintos sectores e instituciones: las regulaciones en torno al trabajo y el empleo, el sistema educativo, las políticas de desarrollo social, salud, infraestructura, etc. (Faur, 2014; Esquivel, Faur y Jelin, 2012, UNRISD, 2016).). Se trató de fortalecer medidas para equilibrar los tiempos destinados al empleo y al cuidado familiar, trasladar parte de los cuidados a otras instituciones y regular la contratación de cuidados por parte de las familias (Blofield y Martínez Franzoni, 2014, entre otras). Ahora bien, en términos generales, con anterioridad a la reforma de la ley de matrimonio civil, este impulso tampoco escapó a la mirada heteronormativa.

El contexto de expansión de derechos de la comunidad LGTTBI permite -exige- ampliar la mirada para analizar, como en un contrapunto, las leyes sobre la constitución familiar y el 
Faur, E. Género, diversidad sexual y conciliación familia - trabajo. Contrapuntos entre el derecho de familia y el derecho laboral. Derecho y Ciencias Sociales. Octubre 2018. № 19. (Las familias y el derecho de las familias a dos años de vigencia del Código Civil y Comercial) Pgs 45-62. ISNN 1852-2971. Instituto de Cultura Jurídica y Maestría en Sociología Jurídica. FCJ y S. UNLP

modo en el cual éstas modificaron (o no) la normativa laboral en relación con las licencias por pater/maternidad.

Siguiendo la distinción realizada por Htun y Weldon (2017), se trata de explorar por un lado, el "derecho de familia", que incluye las reglas sobre el matrimonio y su disolución, así como los derechos, obligaciones y capacidades de los/as cónyuges entre sí y en relación con sus hijos e hijas. Por el otro, las "políticas de familia", que incluyen dispositivos relacionados con los cuidados familiares, como las licencias por maternidad y paternidad, la provisión de servicios de cuidado infantil, y las asignaciones familiares. Mientras que el derecho de familia clasifica el estatus de los individuos que forman parte de las familias, las políticas de familia refieren a la división sexual del trabajo e impactan sobre las relaciones de clase, en la medida que distribuyen prestaciones y beneficios según determinados patrones de titularidad (Htun y Weldon, 2017). Dado el foco de indagación de este artículo, acotaré el análisis al contrapunto entre derecho de familia y derecho laboral y, dentro del último, a las disposiciones en torno a la provisión de licencias para el cuidado de los/as recién nacidos/as.

La perspectiva de Nancy Fraser sobre la justicia social contribuirá al desarrollo del análisis. Según esta autora, las normas relacionadas con el reconocimiento son aquellas que buscan superar la desigualdad cultural, y la consiguiente limitación de derechos a ciertos grupos, mientras que las de redistribución apuntan a reducir las desigualdades socioeconómicas. Ahora bien, el avance normativo en términos de reconocimiento no necesariamente redunda en una mayor redistribución -o viceversa- (Fraser, 1997a). Que el reconocimiento se conecte con un patrón determinado de redistribución depende, en última instancia, de las particularidades de las normas que se analicen (Fraser, 1997b).

Entendemos que tanto el derecho de familia como el derecho laboral parten de una definición acerca de quiénes serán los sujetos titulares de derechos, es decir, del reconocimiento. Desde este enfoque: ¿qué derechos se protegen? ¿Los de quiénes? ¿Se reconoce igualdad de derechos y responsabilidades para sujetos diferentes? Por su parte, cuando la normativa laboral, definen licencias por maternidad y paternidad y servicios de cuidado de niños/as, también incorpora una dimensión de clase, vinculada con la redistribución: ¿De qué modo se asignan los derechos y las prestaciones para el cuidado entre trabajadores según el género? ¿Cómo se incluyen las familias formadas por personas/parejas LGTTBI?

Conceptualmente, se pueden definir dos tipos ideales contrapuestos: uno asentado en la noción de provisión universal y paridad en el cuidado (Fraser, 1997a) en la cual el género sería irrelevante a la hora de establecer derechos y responsabilidades en el terreno productivo 
Faur, E. Género, diversidad sexual y conciliación familia - trabajo. Contrapuntos entre el derecho de familia y el derecho laboral. Derecho y Ciencias Sociales. Octubre 2018. № 19. (Las familias y el derecho de las familias a dos años de vigencia del Código Civil y Comercial) Pgs 45-62. ISNN 1852-2971. Instituto de Cultura Jurídica y Maestría en Sociología Jurídica. FCJ y S. UNLP

y reproductivo. Otro, sostenido en un modelo de familia en el cual las mujeres continúan siendo definidas como principales proveedoras de cuidado, y por tanto, de la armonización de responsabilidades, y que permanece inalterado pese al reconocimiento de los derechos de LGTTBI. En el medio, en tiempos en los que la heteronorma no resulta crucial para la conformación de vínculos familiares, un sinnúmero de posibilidades podría sostener sistemas de corresponsabilidad en los cuidados. Veamos cómo se presentan estas regulaciones en el caso argentino.

\section{Derecho de familia. Un largo proceso hacia la igualdad de género}

El matrimonio civil fue introducido en Argentina en el año 1888 mediante la ley 2393. Previamente, el Código Civil (sancionado en 1869) incluía reglas relacionadas a la familia, muchas de ellas guiadas por los principios católicos (Jelin, 2017) ${ }^{2}$. Durante un largo período, las mujeres estuvieron sujetas a las decisiones de sus maridos en diversos aspectos de sus vidas, mientras que los padres detentaban los derechos sobre sus hijos e hijas (mediante la figura de la patria potestad). En la década de 1980, luego de una larga -y oscura- dictadura militar (1976-1983), los cambios en el derecho de familia se encontraron entre las primeras demandas de los movimientos de mujeres. La ley 23.264 promulgó en 1985 la patria potestad compartida -derechos iguales para ambos padres sobre hijos menores no emancipados- e igualdad de derechos para hijos e hijas de parejas casadas o nacidos a partir de una unión consensual. Apenas después, en 1987, se consagró la Ley de Divorcio vincular (Ley 23.515) (Barrancos, 2017; Jelin, 2017).

En 1994, la reforma de la carta magna de la nación otorgó jerarquía constitucional a los tratados internacionales de derechos humanos (incluida la Convención sobre la Eliminación de Todas las formas de Discriminación contra la Mujer y la Convención sobre los Derechos del Niño). En los años subsiguientes, se promulgó una serie de leyes protectoras, entre éstas: la Ley de Violencia Doméstica (Ley 24.417 de 1996), la Ley de Protección Integral contra la Violencia de Género (Ley 26.485 de 2009) y la Ley de Salud Sexual y Procreación Responsable (25.674 de 2002). Lentamente, las mujeres adquirieron una serie de derechos en el ámbito de las familias y en el terreno de su autonomía personal. A pesar de ello, el debate parlamentario desarrollado en 2018, en relación con la interrupción voluntaria del embarazo,

\footnotetext{
${ }^{2}$ Según estos principios, la diferencia de sexos es una cuestión "natural" y no una relación social definida históricamente, y la familia (en singular) es concebida como una institución sagrada basada en la "unión natural" de un hombre y una mujer. Ver Fundación Comunidad Valenciana (2006)

http://es.catholic.net/op/articulos/10125/doctrina-social-de-la-iglesia-sobre-la-familia.html (22/7/2018)
} 
Faur, E. Género, diversidad sexual y conciliación familia - trabajo. Contrapuntos entre el derecho de familia y el derecho laboral. Derecho y Ciencias Sociales. Octubre 2018. № 19. (Las familias y el derecho de las familias a dos años de vigencia del Código Civil y Comercial) Pgs 45-62. ISNN 1852-2971. Instituto de Cultura Jurídica y Maestría en Sociología Jurídica. FCJ y S. UNLP

dio cuenta de la férrea oposición que aún existe a la hora de ampliar derechos a las mujeres y garantizar su autonomía para decidir sobre sus cuerpos.

En el caso de la población LGTTBI, hasta 2010 las familias formadas por dos madres o dos padres no gozaron de los mismos derechos que las familias constituídas por parejas heterosexuales. Los sujetos titulares de derechos conyugales se definían a partir de un vínculo heterosexual, que excluía cualquier otra manifestación. En el ámbito de la parentalidad, la falta de reconocimiento de parejas del mismo sexo repercutía en que solo a uno de los miembros de la pareja se le reconocía el vínculo legal respecto de sus hijos e hijas (Bacin y Gemetro, 2015).

La Ley de Matrimonio Igualitario (26.618) de 2010 significó un punto de inflexión. Al igualar los derechos civiles de las parejas heterosexuales y homosexuales, dejó atrás la falta de reconocimiento presente en las leyes anteriores. Durante el debate de la ley, la comunidad LGTTBI basó su demanda en el principio de igualdad ("los mismos derechos con los mismos nombres") y encuadró a estos vínculos como "matrimonios igualitarios". De acuerdo con Carlos Fígari, éste fue un aspecto específico del proceso argentino, que constituyó el "factor de su éxito" (Fígari, 2014: 626).

Por su parte, el decreto presidencial de necesidad y urgencia $\mathrm{N}^{\circ}$ 1006/2012 reconoció la igualdad de derechos de las parejas de gays y lesbianas en el ámbito de la parentalidad y no simplemente como cónyuges. Estos avances se consolidaron con la sanción de la ley de Identidad de Género ( $N^{\circ} 26.743$ ) en 2012, que habilitó la modificación de la identidad, en los documentos personales, a fin de respetar el género autopercibido por cada persona. Finalmente, en 2013, la ley 26.862 garantizó el acceso a las técnicas de fertilización asistida a todas las personas mayores de 18 años, sin distinción de estado civil, orientación sexual ni identidad de género.

En 2015, un nuevo Código Civil y Comercial (CCyC) alineó las regulaciones familiares a la perspectiva de derechos humanos, que reconoce el pluralismo y la diversidad de los sujetos titulares de derechos. Para empezar, el CCyC emplea un lenguaje neutro en términos de género, que supera la heteronorma: refiere a "cónyuges" en lugar de "marido y mujer" y "progenitores" en lugar de "madre y padre". Alude, además, a la filiación de una manera neutral, no habla de 'madres', sino de 'la persona que da a luz' -y de este modo reconoce a los varones trans como potenciales gestantes, mientras que se alinea con la ley de identidad de género, basada en la "identidad autopercibida" (Artículo 2, Ley 26.743)-.

El nuevo código busca, también superar la jerarquización masculina de la reglamentación anterior. Mientras la legislación anterior priorizaba el apellido del padre en la identidad de la 
Faur, E. Género, diversidad sexual y conciliación familia - trabajo. Contrapuntos entre el derecho de familia y el derecho laboral. Derecho y Ciencias Sociales. Octubre 2018. № 19. (Las familias y el derecho de las familias a dos años de vigencia del Código Civil y Comercial) Pgs 45-62. ISNN 1852-2971. Instituto de Cultura Jurídica y Maestría en Sociología Jurídica. FCJ y S. UNLP

prole, y colocaba el apellido materno como opcional -pero siempre en un segundo lugar-, el CCyC otorga libertad a los/as progenitores en el momento de decidir el orden de los apellidos de un/a niño/a. (Art. 64) Asimismo, hace explícito que las madres y los padres tienen el mismo deber de dar "atención y dirección a sus hijos", incluso después de un divorcio (Art. 638 y ss). Con ello, deja atrás la noción de patria potestad para sostener el principio de coresponsabilidad parental. De tal modo, el derecho de familia refleja en la Argentina contemporánea una lógica fundada en los principios de derechos humanos, la igualdad y la no discriminación. Reconoce la diversidad de modelos familiares y otorga el mismo estatus a las personas, sujetos de derechos, cualquiera sea su orientación sexual o identidad de género (Herrera et al., en prensa; Jelin, 2017).

Desde un punto de vista legal, la Ley de Matrimonio Igualitario implica que los derechos laborales deben incorporar los cambios filosóficos y de lenguaje impulsados por los derechos civiles y familiares (Herrera et al., en prensa). Sin embargo, más allá de la cuestión del lenguaje, una lógica igualitaria podría promover una mejor redistribución de las tareas entre progenitores, superando la idea de que las mujeres (o quienes gestan) son las principales responsables del cuidado de los niños. ¿Cómo impactó esta transformación en la regulación laboral -en particular en torno a las licencias para cuidar a hijos e hijas de trabajadores/as?

\section{Derecho laboral: la persistencia de una lógica maternalista}

Históricamente, la regulación laboral intentó conciliar las responsabilidades familiares con la participación femenina en la fuerza de trabajo. Este proceso comenzó en Argentina en 1907, y fue tan dinámico como controversial durante las primeras décadas del siglo XX (Nari, 2004). El propósito era armonizar el empleo y la maternidad a través de prohibir despidos de trabajadoras embarazadas, garantizar licencias por maternidad y proveer guarderías en las empresas que emplearan a más de 50 mujeres. Estas medidas resultaron un gran avance en la protección de derechos de las mujeres trabajadoras, cuya maternidad se encontraba totalmente desprotegida. Al mismo tiempo, las normas reforzaron el "modelo familiar de varón proveedor y mujer ama de casa", al asignar diferentes responsabilidades y derechos a hombres y mujeres. Los hombres, en su carácter de "jefes de hogar" accedían a asignaciones familiares, seguros de salud y previsión social, mientras que las mujeres titularizaban derechos relacionados con su rol de madres y principales proveedoras de cuidado. Los derechos de las mujeres se forjaron a partir un patrón maternalista (Nari, 2004) y no como derechos de ciudadanía. 
Faur, E. Género, diversidad sexual y conciliación familia - trabajo. Contrapuntos entre el derecho de familia y el derecho laboral. Derecho y Ciencias Sociales. Octubre 2018. № 19. (Las familias y el derecho de las familias a dos años de vigencia del Código Civil y Comercial) Pgs 45-62. ISNN 1852-2971. Instituto de Cultura Jurídica y Maestría en Sociología Jurídica. FCJ y S. UNLP

Si bien es claro que el embarazo y los primeros meses después de la llegada de un/a bebé, las mujeres (y personas gestantes según el lenguaje del $\mathrm{CCyC}$ ) requieren de protección especial, pasado este período las regulaciones podrían definir similares derechos y responsabilidades a ambos progenitores. La Organización Internacional del Trabajo (OIT) a través del Acuerdo 198 del 2000, consideró que el mínimo de licencias maternales se debe establecer en 14 semanas. Blofield y Martínez Franzoni (2014) denominan a esta propuesta como el "piso maternalista" de las políticas conciliatorias, que distinguen de las políticas netamente maternalistas como aquellas que exaltan el papel de las madres sin buscar reducir las brechas de género en los cuidados.

En los países más innovadores en esta materia -como Suecia, Noruega y Dinamarca-, las licencias aplicadas han apostado por un sistema intercambiable, en el cual, pasado un primer período de protección a la persona gestante, los progenitores pueden optar de qué manera distribuirán las licencias para el cuidado de sus hijos/as. Adicionalmente, se han aplicado incentivos para que los padres asuman esta tarea, por ejemplo, mediante un sistema de "cuotas paternas", exenciones impositivas, etc. (Haas y Rostgaard, 2011).

En el caso argentino, desde 1976, la Ley de Contrato de Trabajo (LCT) válida para el sector privado, otorga 90 días corridos como licencia por maternidad. Por su parte, los hombres empleados sólo cuentan con dos días (Art. 177, LCT № 20.744). En más de cuarenta años, no se ha logrado expandir estos permisos parentales. Sin embargo, existe una importante heterogeneidad entre jurisdicciones, sectores y ramas de ocupación. ${ }^{3}$

En el ámbito del empleo público, quienes trabajan en la administración nacional gozan de mejores condiciones que aquellos/as que se vinculan al sector privado. Según el Decreto 214/2006, sus licencias por maternidad se establecen en 100 días y por paternidad, en 5 días. Aún así, en el contexto de un sistema federal, cada jurisdicción define sus propias normativas respecto del empleo público. Hasta el año 2017, sólo algunas provinicas y municipalidades extendieron el plazo de licencia por maternidad. La Rioja contempla uno de los esquemas más amplios en términos de licencias (210 días para las madres y 30 para los padres, según la ley 8164). Mientras tanto, en la mayoría de las provincias, la extensión de las licencias no alcanza siquiera a cubrir el estándar mínimo propuesto por la OIT (Díaz Langou y Florito, 2017). De

\footnotetext{
${ }^{3}$ La ley de Contrato de Trabajo es aplicable a los trabajadores asalariados con excepción de los empleados de la administración pública nacional, provincial y municipal y de los/as docentes, quienes están cubiertos por un estatuto especial. La ley de 1999 de Empleo Público Nacional n 24164 y el decreto 214/2006 regulan el empleo en el sector público a nivel nacional. Los empleados públicos de diferentes jurisdicciones son gobernados por leyes particulares, y ello no es distinto en materia de educación, donde las normas son diferentes en cada provincia. La totalidad de regulaciones laborales es complementada por convenios a los cuales se arriba luego de negociaciones colectivas.
} 
Faur, E. Género, diversidad sexual y conciliación familia - trabajo. Contrapuntos entre el derecho de familia y el derecho laboral. Derecho y Ciencias Sociales. Octubre 2018. № 19. (Las familias y el derecho de las familias a dos años de vigencia del Código Civil y Comercial) Pgs 45-62. ISNN 1852-2971. Instituto de Cultura Jurídica y Maestría en Sociología Jurídica. FCJ y S. UNLP

las 24 jurisdicciones argentinas, sólo una -Tierra del Fuego- tiene un esquema de responsabilidad compartida. En este caso, las mujeres gozan de una licencia de 30 días, mientras que los hombres pueden ausentarse de su trabajo durante los 15 posteriores al nacimiento. Pasado este período, se otorgan 180 días de licencia que pueden dividirse entre ambos progenitores, según su propia decisión. En definitiva, cuando se revisan las regulaciones en torno al cuidado y las licencias otorgadas a madres y padres, la diversidad constituye la norma (Faur, 2014). Adicionalmente, algunos/as trabajadores/as del sector público han conseguido licencias más extensas a partir de las negociaciones colectivas (es el caso, por ejemplo, de los/as empleados judiciales y de los/as docentes).

Desde una perspectiva de género, la legislación argentina refleja dos cuestiones principales. En primer lugar, la protección está basada en una lógica de diferenciación de dispositivos y titularidad de derechos entre varones y mujeres. Son las mujeres quienes -en mayor medida- acceden a derechos como cuidadoras. La licencia por paternidad es escasa en todos los casos, lo que exime a los varones de las responsabilidades de cuidado familiar. En definitiva, si la legislación laboral buscó "conciliar" las responsabilidades familiares y laborales, lo hizo a partir de definir un sujeto femenino como titular de derechos $\mathrm{y}$ responsabilidades (Faur, 2006). Así, mientras el derecho de familia introdujo un lenguaje neutro en términos de género e iguales responsabilidades parentales, las políticas de familia continúan siendo específicas en términos de género.

El tema está en discusión, pero los avances son lentos e improbables. En mayo de 2017, la Cámara de Diputados contaba con 43 proyectos de ley suscriptos por casi un centenar de legisladores. Más de la mitad de las propuestas incluían la extensión de la licencia de maternidad y paternidad. Algunos legisladores reconocieron que los debates sobre este tema quedaban truncados por el costo adicional que la extensión de licencias supondrían para las empresas y para el estado. En ocasiones, se argumentó, incluso que extender la licencia paterna podría tener un impacto negativo en el empleo masculino, e incrementaría su participación en el sector informal. Lo que se esconde detrás de argumentos de este tipo es que no se busca igualar condiciones para los distintos sujetos sino privilegiar el empleo masculino. Se trata de un nudo crítico para la igualdad de género, que también tiene implicancias en la perpetuación de desigualdades de clase.

A finales del año 2017, el Poder Ejecutivo presentó un proyecto de reforma laboral que supondría el incremento de la licencia para padres vinculados con el sector privado, estableciendo su licencia en 15 días corridos. Se trata, en definitiva, de un cambio parcial, que 
Faur, E. Género, diversidad sexual y conciliación familia - trabajo. Contrapuntos entre el derecho de familia y el derecho laboral. Derecho y Ciencias Sociales. Octubre 2018. № 19. (Las familias y el derecho de las familias a dos años de vigencia del Código Civil y Comercial) Pgs 45-62. ISNN 1852-2971. Instituto de Cultura Jurídica y Maestría en Sociología Jurídica. FCJ y S. UNLP

procura extender los días de vinculación paterna sin consolidar un nuevo marco conceptual igualitario e intercambiable- en relación con los cuidados. De tal modo, el proyecto se aleja de una visión de paridad en el cuidado y no incorpora la nueva concepción de vínculos familiares y parentales según el CCyC. Además, presenta la paradoja de formar parte de un proyecto de reforma laboral que restringe derechos para los trabajadores/as (por ejemplo, en relación con la indemnización por despido).

Desde el punto de vista de la diversidad, este ordenamiento responde a un carácter binario (basado en la diferenciación entre hombres y mujeres y excluyendo otras identidades). A pesar de los avances en los derechos civiles, las regulaciones laborales muestran un notorio retraso en el reconocimieno de los derechos LGTTBI, pues omiten la consideración de las parejas formadas por personas del mismo sexo/género en el ordenamiento de las licencias para el cuidado infantil. De tal modo, la heteronormatividad persiste como canon prevaleciente. $^{4}$

Sólo en algunos sectores -de hecho, en los más protegidos, como el sector judicial-, se observan cambios, mayormente alcanzados mediante negociaciones colectivas. En 2015, se aprobó un Convenio Colectivo de Trabajo para integrantes del Poder Judicial y de los Ministerio Públicos de la CABA que entre otras cosas, establece la licencia por maternidad en 120 días (art. 48) y la de paternidad y co-maternidad (para la madre no gestante) en 60 días (art. 49). ${ }^{5}$

En el caso del Ministerio Público Fiscal, se aprobó un nuevo régimen de licencias parentales que incorpora avances en materia de diversidad sexual. La nueva regulación modificó su lenguaje, adecuándolo al del CCyC. No refiere a "licencia por paternidad" o por "maternidad", sino a "licencia por nacimiento o adopción de hijos/as". A los progenitores, los denomina "persona gestante" y "progenitor no gestante" (Resolución N 3140/16, Art. 4). También extiende la licencia a 110 días por el nacimiento en el caso de quienes llevan adelante el embarazo y parto, y a 15 en el caso de no gestantes. Además, la licencia por adopción se amplía para los progenitores solteros o parejas formadas por dos varones. ${ }^{6} \mathrm{Se}$ trata de avances profundos en su filosofía, en la medida que deconstruyen los presupuestos de género y diluyen la lógica heteronormativa. A pesar de ello, por una parte, las licencias continúan siendo breves, y no alcanzan a cubrir el piso fijado por la OIT -establecida en 14

\footnotetext{
${ }^{4}$ Algunas organizaciones de la sociedad civil, como CIPPEC y ELA, junto con UNICEF, se han avocado a estos derechos enmarcados como "licencia universal" y han buscado poder superar al heternormativismo (Diaz Langou y Florito, 2017).

${ }^{5}$ Fuente: convenio colectivo de trabajo del Poder Judicial de la Ciudad de Buenos aires 2015.

${ }^{6}$ Fuente: Procuración General de la Nación. Resolución No 3140/16.
} 
Faur, E. Género, diversidad sexual y conciliación familia - trabajo. Contrapuntos entre el derecho de familia y el derecho laboral. Derecho y Ciencias Sociales. Octubre 2018. № 19. (Las familias y el derecho de las familias a dos años de vigencia del Código Civil y Comercial) Pgs 45-62. ISNN 1852-2971. Instituto de Cultura Jurídica y Maestría en Sociología Jurídica. FCJ y S. UNLP

semanas-. Por otra, mantienen la asignación principal de responsabilidades de cuidado a la persona que gesta.

El vacío legal y regulatorio existente en ocasiones impulsa a parejas homosexuales a presentar recursos de amparo para obtener derechos. En la provincia de Buenos Aires, a partir de un reclamo administrativo realizado por una pareja de mujeres -ambas empleadas judiciales-, la Suprema Corte de Justicia, mediante el Acuerdo 3875 de 2017 modificó los términos de las licencias para el personal del poder judicial de dicha provincia. El reclamo se asentó en la consideración que ambas se corresponsabilizaron de la lactancia de su hija y buscaban ampliar la licencia para la madre no gestante ${ }^{7}$. La flamante acordada buscó alinear las licencias con los principios de no discriminación e igualdad que protegen los derechos de toda persona humana, "circunstancia que determina la supresión de cualquier referencia al género de la persona involucrada" (Art. VI). Asimismo, en la provincia de Río Negro, una docente -madre no gestante- logró acceder a licencia por maternidad a partir de una decisión administrativa. Los casos son sin duda variados y una investigación ad-hoc permitiría avanzar en la producción de conocimiento y la sistematización de antecedentes.

En general, cuando se logra ampliar derechos, por el momento, se originan en demandas específicas y se corresponden con los sectores de ocupación que cuentan con protecciones laborales más extendidas. Estos casos resultan precedentes que mientras se resuelven en la jurisdicción y sector de ocupación de los/as trabajadores/as que inician los reclamos, ponen en evidencia los vacíos existentes en el régimen de licencias para adecuarse al nuevo marco legal en materia de diversidad sexual.

Finalmente, hay consideraciones de clase que requieren señalarse. Limitadas como son, las normas laborales ni siquiera protegen a todos/as los/as trabajadores/as, sino que dejan fuera de su égida a aquellos/as empleados/as de manera informal, como asi también a los/as trabajadores/as independientes (Faur, 2014). Las mujeres asalariadas son quienes más ocupan los empleos informales y, en consecuencia, quienes menos acceden a estos beneficios (PNUD, 2014). La protección limitada de licencias y la escasez de servicios de cuidado termina generando una menor participación en el mercado laboral por parte de las mujeres de clases populares sin capacidad de contratar servicios que el estado no provee (Faur y Pereyra, 2018).

El escenario resultante es uno de complejas interacciones entre las regulaciones estatales, los mercados, y las familias, que impactan tanto desde el punto de vista de género como también en términos de clase.

\footnotetext{
${ }^{7}$ El reclamo fue patrocinado por el programa de extensión de "Diversidad sexual y derecho de familia" de la Facultad de Ciencias Jurídicas de la Universidad Nacional de La Plata.
} 
Faur, E. Género, diversidad sexual y conciliación familia - trabajo. Contrapuntos entre el derecho de familia y el derecho laboral. Derecho y Ciencias Sociales. Octubre 2018. № 19. (Las familias y el derecho de las familias a dos años de vigencia del Código Civil y Comercial) Pgs 45-62. ISNN 1852-2971. Instituto de Cultura Jurídica y Maestría en Sociología Jurídica. FCJ y S. UNLP

\section{Consideraciones finales}

Este artículo examinó de qué manera la ampliación de derechos para la comunidad LGTTBI impactó en las políticas que asignan la responsabilidad por el cuidado infantil a padres y madres trabajadores/as. El panorama da cuenta de marcados contrastes y de cambios puntuales, incipientes.

El reconocimiento de derechos para la población LGTTBI coexiste con la sombra maternalista que aún pende sobre las regulaciones laborales que establecen licencias para el cuidado infantil. La igualdad de derechos aún no se ha logrado institucionalizar de manera plena, dando cuenta que a pesar de que el matrimonio es igualitario, las responsabilidades del cuidado, de acuerdo con las normativas legales, aún no lo son.

El resultado es la coexistencia de lógicas diferentes en las políticas estatales, que dan cuenta de las "muchas manos" que tiene el Estado a la hora de regular normas que impactan las relaciones de género. El derecho de familia, de manera creciente, refleja la lógica de derechos y el reconocimiento de las múltiples identidades de género. Mientras tanto, el derecho laboral se erige sobre la lógica del maternalismo, que distingue roles y responsabilidades entre géneros y enfatiza el sujeto femenino como el titular de la conciliación de responsabilidades de empleo y cuidado familiar. Aún así, las prestaciones son breves e incompletas y no facilitan una eficaz redistribución de cuidados entre géneros ni entre las familias, los estados y los mercados. Así, se refuerzan, además, las ya agudas desigualdades socioeconómicas.

En definitiva, mientras el derecho de familia avanzó en el reconocimiento de derechos, el derecho laboral no incorporó las modificaciones de la normativa civil, y se encuentra lejos de establecer un patrón que se encamine hacia la paridad de los cuidados.

Entre las complejidades de este escenario, se debe apuntar que se trata de políticas que requieren intervenciones diferentes por parte de los distintos niveles y jurisdicciones estatales. Los avances en el derecho de familia son filosófica y culturalmente significativos, al incluir a sujetos excluídos dentro de un sistema institucional, pero no exigen inversiones presupuestarias de envergadura: las instituciones que los protegen -registros civiles, juzgados de familia, etc.- preexisten a las normas que reconocieron derechos a la población LGTTBI. En cambio, las políticas redistributivas en materia de cuidado, y en particular, las referidas a las licencias y servicios de atención infantil, requieren movilizar nuevos recursos, así como una inversión sostenida por parte del estado y del mercado para poder ampliar derechos. 
Faur, E. Género, diversidad sexual y conciliación familia - trabajo. Contrapuntos entre el derecho de familia y el derecho laboral. Derecho y Ciencias Sociales. Octubre 2018. № 19. (Las familias y el derecho de las familias a dos años de vigencia del Código Civil y Comercial) Pgs 45-62. ISNN 1852-2971. Instituto de Cultura Jurídica y Maestría en Sociología Jurídica. FCJ y S. UNLP

¿Cuáles son los desafíos pendientes en el caso argentino? Primero, se requieren políticas de cuidado integrales que se adapten a los avances que, en término de reconocimiento, han logrado establecerse en el derecho de familia. Además, se deben promover permisos de paternidad y maternidad (o de parentalidad) equivalentes, y cuotas intransferibles. Este objetivo no solo permite una corresponsabilidad masculina en las tareas de crianza, sino que además, contribuiría a eliminar los prejuicios respecto del costo de la maternidad en el mercado laboral, y apuntaría a otorgar a la reproducción social una mayor jerarquía social y comunitaria, sin que quede subsumida a responsabilidades individuales o familiares (Comas d’Argemir, 2017).

Al mismo tiempo, en la region más desigual del mundo, la necesidad de cerrar las brechas socioeconómicas resulta crucial (Esquivel, 2011; Rico \& Robles, 2016). Las políticas relacionadas con la legislación laboral necesitan ampliar sus prestaciones y mejorar el patrón de redistribución para impactar en una mayor igualdad de clase y género, que supere la visión maternalista y avance hacia un sistema de doble provisión y paridad en el cuidado (Fraser, 1997a). Ello supondrá el ajuste de los tiempos de trabajo establecidos en la jornada laboral, la implementación del principio de equidad en derechos de hombres y mujeres y la extensión de la licencia por parentalidad y de servicios de cuidado para todos/as los/as trabajadores/as (con independencia de su sexo o identidad de género).

Cimentar respuestas efectivas frente a estos desafíos requerirá, en última instancia, de un profundo cambio cultural y de una intervención (e inversión) activa del Estado, que movilice la coordinación de un conjunto de actores e instituciones y la corresponsabilidad de los mercados. En el año 2018, en un contexto de creciente endeudamiento externo y ajuste fiscal, las perspectivas de consolidación de estas iniciativas son, por lo menos, inciertas.

\section{Referencias bibliográficas}

Bacin, G y Gemetro, F (2015), Reconocimiento igualitario de NNA y niñas defamilias comaternales, en Dossier Derechos Humanos, Año 4 No. 10, Sistema Argentino de Información Jurídica. Ministerio de Justicia y Derechos Humanos. Buenos Aires, INFOJUS. 71-79. Junio.

Barrancos D (2007), Mujeres en la sociedad argentina: una historia de cinco siglos, Buenos Aires, Sudamericana.

Barrancos D (2017) "Feminismos y agencias de las sexualidades disidentes", en Faur E (ed) Mujeres y varones en la Argentina de hoy. Géneros en movimiento. Buenos Aires, Siglo XXI Editores. Pp 29-50. 
Faur, E. Género, diversidad sexual y conciliación familia - trabajo. Contrapuntos entre el derecho de familia y el derecho laboral. Derecho y Ciencias Sociales. Octubre 2018. № 19. (Las familias y el derecho de las familias a dos años de vigencia del Código Civil y Comercial) Pgs 45-62. ISNN 1852-2971. Instituto de Cultura Jurídica y Maestría en Sociología Jurídica. FCJ y S. UNLP

Binstock, G. (2018), "Hogares y organización familiar". En: Piovani, J. I. y Salvia, A. (coords) La Argentina en el siglo XXI. Cómo somos, vivimos y convivimos en una sociedad desigual. Bs. As.: Siglo XXI, pp. 421-442

Blofield M y Martínez Franzoni J (2014), 'Maternalism, Co-responsibility and Social Equity: a Typology of Work-family Policies', Social Politics: International Studies in Gender, State \& Society 22(1). Pp 38-59.

Connell RW (1995), Masculinities. Berkeley: University of California Press.

Creighton C (1999), The rise and decline of the 'male breadwinner family' in Britain. In: 'Special Issue on the Family', Cambridge Journal of Economics, 23(5).

Crompton R (ed) (1999), Restructuring Gender Relations and Employment. The Decline of the Male Breadwinner. New York: Oxford University Press.

Crompton R (2006), Employment and the Family. The reconfiguration of work and family life in contemporary societies. London: Cambridge University Press.

Díaz Langou G y Florito J (2017), ¿Cómo promover un cambio en el régimen de licencias por maternidad, paternidad y familiares? Lecciones aprendidas del análisis de casos provinciales. Disponible en: www.cippec.org/wp-content/uploads/2017/03/1071.pdf (última consulta el 15 de agosto de 2017).

Esquivel, V (2011), La economía del cuidado en América latina. Poniendo a los cuidados en el centro de la agenda. Panamá, PNUD

Esquivel V, Faur E y Jelin E (2012), Las lógicas del cuidado infantil. Entre las familias, el estado y el mercado. Buenos Aires. , IDES-UNFPA-UNICEF.

Faur E (2006), 'Género y conciliación familia-trabajo. Legislación laboral y subjetividades masculinas en América Latina, en L. Mora y M. J. Moreno (eds) Cohesión social, políticas conciliatorias y presupuesto público. Una perspectiva de género, pp. 127-50. Mexico DF: Fondo de Población de las Naciones Unidas--GTZ.

Faur E (2011), A Widening Gap? The Political and Social Organization of Childcare in Argentina. Development \& Change, 42(4) 967-94.

Faur E (2014), El cuidado infantil en el siglo XXI. Mujeres malabaristas en una sociedad desigual. Buenos Aires: Siglo XXI editores.

Faur, E y Pereyra, F (2018), “Gramáticas del cuidado”, en Piovani, J. I. y Salvia, A. (coords) La Argentina en el siglo XXI. Cómo somos, vivimos y convivimos en una sociedad desigual. Bs. As.: Siglo XXI.

Fígari C (2014), Queer Argie, American Quarterly, 66(3) September, 621-31. 
Faur, E. Género, diversidad sexual y conciliación familia - trabajo. Contrapuntos entre el derecho de familia y el derecho laboral. Derecho y Ciencias Sociales. Octubre 2018. № 19. (Las familias y el derecho de las familias a dos años de vigencia del Código Civil y Comercial) Pgs 45-62. ISNN 1852-2971. Instituto de Cultura Jurídica y Maestría en Sociología Jurídica. FCJ y S. UNLP

Fraser N (1997a), Iustitia Interrupta. Reflexiones críticas desde la posición "post-socialista”. Bogotá: Siglo del Hombre Editores - Universidad de Los Andes.

Fraser N (1997b), 'Heterosexism, Misrecognition, and Capitalism: A Response to Judith Butler', Social Text 52-3 (Fall/Winter) 279-89.

Haas, L y Rostgaard, T (2011), Fathers' rights to paid parental leave in the Nordic countries: consequences for the gendered division of leave, Community, Work \& Family, 14:2, 177 195, DOI: $10.1080 / 13668803.2011 .571398$

Herrera M, de la Torre N y Silva SA (en prensa), "Impacto de las relaciones de familia del Código Civil y Comercial en el Derecho del Trabajo", incluido en Ojeda RH (ed) Tratado Práctico de Derecho del Trabajo (Título II "Relaciones Individuales del Trabajo) Buenos Aires: Editorial Errejus.

Htun M y Weldon L (2017), "States and Gender Justice”, en Morgan, K. J. y Orloff, A. S. (eds) The Many Hands of the State. Theorizing Political Authority and Social Control, New York, Cambridge University Press.

Htun, M y Weldon, L (2010), "When Do Governments Promote Women's Rights? A Framework for the Analysis of Sex Equality Policy. Perspectives on Politics - March 2010 Vol. 8/No. 1. Pp. 207-216.

ILO (2015) Orgullo (Pride) en el trabajo. Un estudio sobre la discriminación en el trabajo por motivos de orientación sexual e identidad de género en Argentina. ILO, Geneva.

INADI (2015) Hacia una redistribución igualitaria de las tareas de cuidado. Buenos Aires, INADI, Secretaría de Derechos Humanos.

Jelin E (2010), Pan y afectos. La transformación de las familias. Buenos Aires: Fondo de Cultura Económica.

Jelin E (2017), "Familia. Un modelo para desarmar", en Faur E (ed) (2017) Mujeres y varones en la Argentina de hoy. Géneros en movimiento. Buenos Aires, Siglo XXI Editores. Pp. 51-73.

Lamas M (2002), Cuerpo: diferencia sexual y género. México: Editorial Taurus

Lind, A y Share, J (2003), Queering development: institutionalized heterosexuality indevelopment theory, practice and politics in Latin America, en: Feminist futures: reimagining women, culture and development, editado por Kum-Kum Bhavnani, John Foran y Priya A. Kurian. Londres, Zed Books, 2003. 55-73

Laya A (2014), El Derecho al Cuidado en los Convenios Colectivos de Trabajo del Sector Privado. Análisis comparativo de Convenios Colectivos en las ramas de actividades con mayor índice de feminización del sector privado. Buenos Aires: ADC-CIEPP-ELA. 
Faur, E. Género, diversidad sexual y conciliación familia - trabajo. Contrapuntos entre el derecho de familia y el derecho laboral. Derecho y Ciencias Sociales. Octubre 2018. № 19. (Las familias y el derecho de las familias a dos años de vigencia del Código Civil y Comercial) Pgs 45-62. ISNN 1852-2971. Instituto de Cultura Jurídica y Maestría en Sociología Jurídica. FCJ y S. UNLP

Martínez Franzoni, J (2008), "Régimen de bienestar familiarista”, en Martínez Franzoni, J ¿Arañando bienestar? Trabajo remunerado, protección social y familias en America Central. Buenos Aires: CLACSO, Consejo Latinoamericano de Ciencias Sociales.

Morgan KJ y Orloff AS (2017), "Introduction: The Many Hands of the State", in Morgan KJ y Orloff AS (eds) The Many Hands of the State. Theorizing Political Authority and Social Control, New York, Cambridge University Press.

Nari M (2004), Políticas de maternidad y maternalismo político. Buenos Aires, Editorial Biblos.

PNUD (2014), Género en el trabajo. Brechas en el acceso a puestos de decisión, Aportes para el desarrollo humano en Argentina, 8, Buenos Aires, PNUD.

Pecheny, M., y de la Dehesa, R. (2010), Sexualidades y políticas en América Latina: el matrimonio igualitario en contexto. en L. Clérico, \& M. Aldao (Ed.) Matrimonio Igualitario en Argentina. Buenos Aires. EUDEBA. Pp. 7-58

Rico N y Robles C (2016), Políticas de cuidado en América Latina. Forjando la igualdad. Santiago de Chile, CEPAL-Cooperación Alemana.

UNRISD (2016) Care Policies: Realizing their Transformative Potential, in Policy Innovations for Transformative Change: UNRISD Flagship Report 2016. Ginebra.

Wainerman, C (2003), "Familia y trabajo. La reestructuración de las fronteras de género", in Wainerman, C (ed.), Familia, trabajo y género. Un mundo de nuevas relaciones, Buenos Aires, UNICEF-Fondo de Cultura Económica. 